\title{
Fuzzy Based Enhanced Smart Rest Room Automated Faucet System
}

\author{
Akazue I. Maureen ${ }^{\mathrm{a}}$, Ajenaghughrure Ighoyota Ben ${ }^{\mathrm{b}}$ \\ ${ }^{a}$ Department of computer science, Delta State University,P.M.B 1, Abraka, Nigeria \\ ${ }^{b}$ Department of computer science, Vels University, Old pallavaram, chennai-600117, India
}

\begin{abstract}
Existing smart rest room automated faucet systems operate on traditional control theory, with absolute control value, irrespective of its degree of accuracy. For example, a hands-free hand wash basin may be programmed to release water when an object is about $2 \mathrm{~cm}$ close to the infrared sensor. This absolute control value has pitfalls such as, ascertaining the quantity of water required for each operation, without activating the water flow switch to release water at maximum rate, leading to wastage of water resources. In order to develop an efficient and accurate smart rest room automated faucet system that will release water at an approximate quantity as required for varying condition, this research paper delves into employing a data-centric model for understanding and designing a smart rest room automated faucet system, that is more accurate in operation and properly utilizes water resources. To achieve this goal, we first designed a fuzzy model using MATLAB, for the proposed smart rest room automated faucet system, and then implemented the model on embedded atmel328 microcontroller, interfaced with an infrared obstacle sensor and an electronic flow control switch to automate rest room faucet activities. In order to understand and improve its operation, data logged from the system infrared sensor for $3 \mathrm{~cm}$ distance, was mined for proper understanding of the system operational accuracy. During which the result indicated proper water utilization at various rate of water dispensation, as a function the nearness of the object to the sensor. This supports the improvement promised by the proposed system, when adopted in existing smart rest room automated faucet systems design and re-design.
\end{abstract}

Index Terms: Smart rest room, automated faucet system, Fuzzy model, water, microcontroller

(C) 2017 Published by MECS Publisher. Selection and/or peer review under responsibility of the Research Association of Modern Education and Computer Science.

\section{Introduction}

Smart rest room automation has received significant boost from researchers involved in smart homes and smart city projects, as well as industries developing commercial products deployed in today's homes, transport systems (vehicle, aeroplanes etc) and lots more places. Primarily for improving healthy living and reduced water consumption. Improving health, which is one of the major aim of smart restroom automated faucet

* Corresponding author. Tel.:

E-mail address: 
system, [3] described a user friendly rest room for elderly and disabled in Europe. A friendly rest room project partly funded by EU through quality of life program, aims at creating a a more user friendly rest room for elderly and disabled person as well as gain more insight into their needs in rest room. In addition, [4] reported a research project that aims at increasing hand washing habit, towards reducing infection transmitted as a result of not washing hands after rest room usage which is usually caused by the inconveniencies of operating faucet. This was achieved through provision of multimedia incentives that attracts people to wash their hands on the smart interactive faucet system, which displays news update and some more entertaining lights flashing in different pattern. Also, [8] proposed and designed an automated urinal flushing system, that automatically flushes after urinating, hence avoid irritating odor and possible infections resulting from user forgetting to flush after use. In addition, [9] developed a purity activated automated toilet flushing system, that monitors the toilet cleanliness with a ph sensor and controls the flushing with a microcontroller

Also, Conservation of water resources being another major motivation behind the development of smart rest room automated faucet systems, Trupti et al[1] designed an automated faucet system to aid in water resource efficient utilization, preventing wastage, and reducing time required to operate two separate faucet controlling supply of hot and cold water for bath. Thereby improving users bath experience, through smart rest approach. The system is cost and energy effective. In addition, [7] developed automated water consumption per capital alert system that notifies users if their water usage is becoming excessive. Furthermore, although not in the context of rest room, but in agriculture, [5] to developed a water flow meter system for plant irrigation. In addition, [2] designed an all-in-one hand-free hand washer, which automatically rinse the hands without any touch, solely promoting hand washing culture and reduce the spread of germs related to improper hand washing. Also [8] proposed and designed an automated urinal flushing system that automatically flushes after urinating, hence avoid irritating odor and possible infections resulting from user forgetting to flush after use

Unfortunately, All these systems developed so far, mainly focuses on water resource conservation and improved health, without proper implementation measures to ensure water resource conservation, since they use traditional control theory, with absolute control value, irrespective of its degree of accuracy, for activation of water flow switch, which is incompetent in determining the approximate amount of water needed per operational state. For example, a hands-free hand wash basin may be programmed to release water when an object is about $2 \mathrm{~cm}$ close to the infrared sensor. This absolute control value falls short in ascertaining amount of water required. In order to develop an efficient and more accurate smart rest room automated faucet system that will release water at an approximate quantity as required for varying condition. A fuzzy model was developed, Splitting the sensor acceptable trigger value into five groups, lower bound, lower upper bound, midrange, upper lower bound, and upper bound. Where lower bound is the minimum sensor acceptable trigger value, upper lower bound is the sensor value right before mid-range value greater than lower bound value, midrange is the average sensor value, lower upper bound is the sensor value right after the mid-range value but not greater than or equal to upper bound value respectively. Each of these groups can be a range of values as well. In order to separate these groups without any crisp boundary our fuzzy rule will be as follows
If value $=$ lower bound then slower flow
If value =higher lower bound then slow
If value $=$ mid range value. then average flow
If value=lower upper bound then fast flow
If value = upper bound then faster flow

Where lowerbound, upper lowerbound, mid-range, lower upper bound, and upper bound are all premise, while slower flow, slow flow, average flow, fast flow and faster flow are all the corresponding consequence of each premise for the system. 


\section{Smart Rest Room Automated Faucet System Fuzzy Model Design}

The FIS model in fig1 below is the architecture of the smart rest room automated faucet system fuzzy model, which simulates the our proposed enhanced smart rest room automated faucet system. It is a single-input single-output model. Where the input variable corresponds to infrared obstacle sensor values in practical implementation. And the output corresponds the electronic flow switch status. The input variable consist of five membership functions, each having a set of values corresponding to the five sub-groups of acceptable sensor value ranges that we mentioned earlier in section 1.0 as the premise(lower bound, upper lower bound, midrange, lower upper bound and upper bound). While the output variable membership functions corresponds to the five consequence slower flow, slow flow, average flow, fast flow and faster flow respectively. Both input and output membership functions mapped to each other through the fuzzy rule we will be discussing latter on.

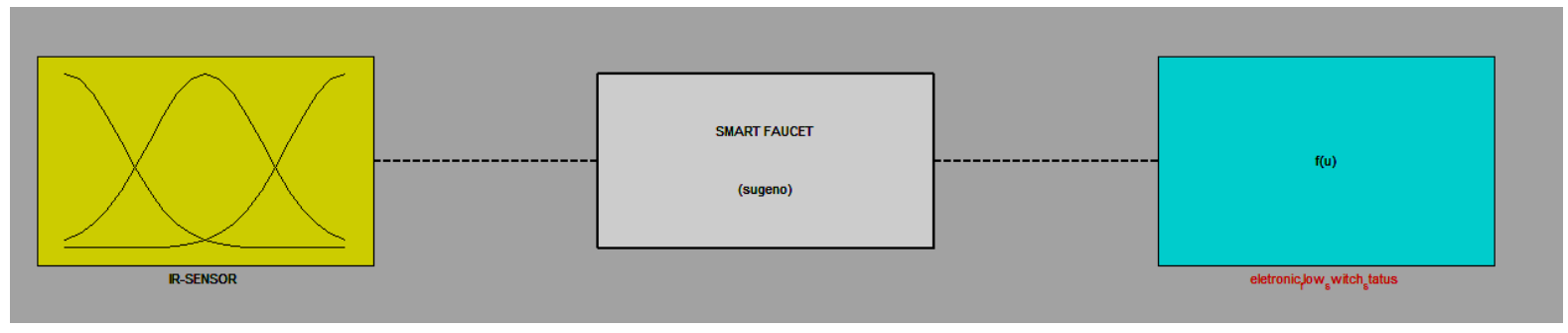

Fig.1. Fuzzy FIS Smart Faucet Model for Rest Room Automation

\section{A. Fuzzy rule}

The fuzzy rule for the FIS madani system model in fig1 above, consist of five(5) linguistics variables, for each input and output variable membership functions. The input variable membership functions linguistics are lowerbound, upper lowerbound, mid-range, lower upper bound, and upper bound. While the output variable membership functions linguistics are slower flow, slow flow, average flow, fast flow and faster flow. Rule formulation here involves directly paring one input membership function linguistics to exactly one corresponding output membership function linguistics variable using the fuzzy "IF.. THEN" rule formation technique. Since we have five each for both input and output variable membership functions, the total rule for the fuzzy FIS PID controller model in fig 1 above will be 5 in total, as detailed below

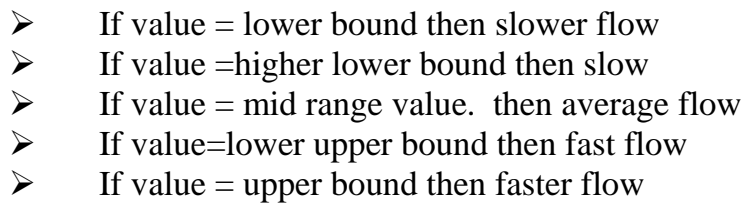

Fig2 below is a fuzzy FIS rule viewer, showing input variable membership function mid-range and its corresponding output variable membership average flow clearly. Hence at this point the smart rest room automated faucet system is activated with water flowing on average speed. Furthermore, we can infer that all other input variable membership function are blank except the mid-range input variable membership function. Also, the output membership functions are all blank, except for the average flow membership function output variable. Therefore, this confirms the effectiveness of our proposed enhanced smart rest room automated faucet system. 


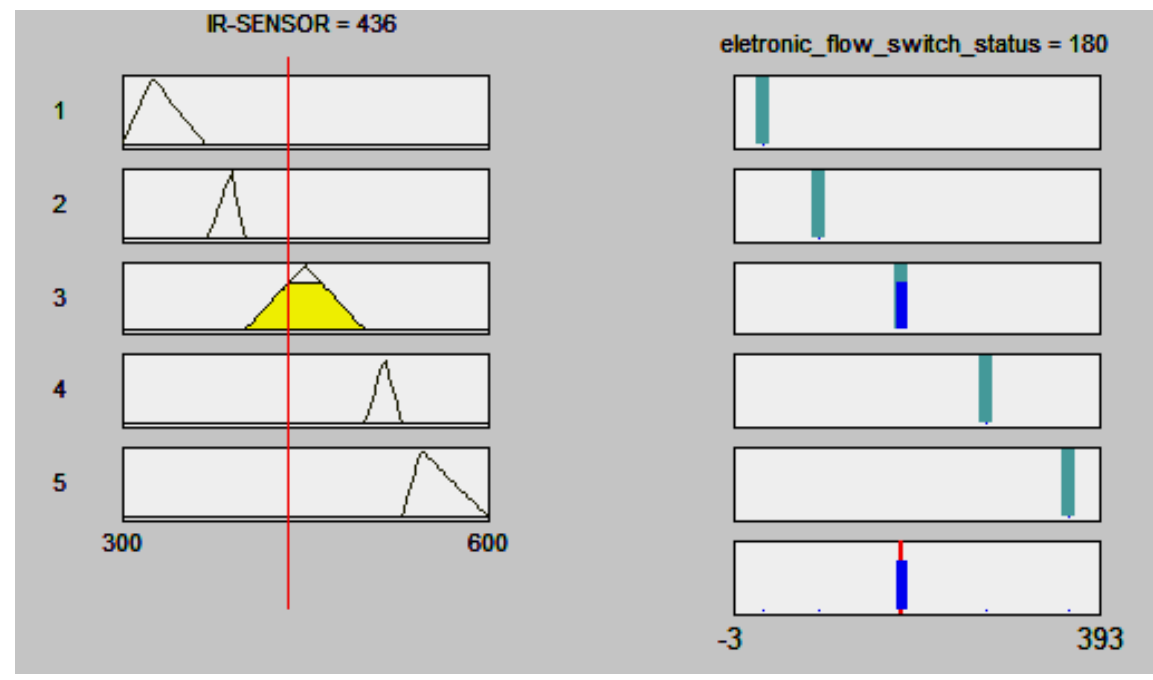

Fig.2. Matlab Screen Shot of the Fuzzy Based Smart Rest Room Automated Faucet System Fuzzy Model Rule Viewer

\section{B. Membership functions}

\section{INPUT}

The membership function for each input variable corresponding to obstacle sensor values, which are related to object distance in practical implementation, are range of values that is further divided into five sub groups(lower bound, upper lowerbound, mid-range, lower upper bound, upper bound) in our system to ensure effective and accurate operation of the system. As shown in fig3. They are all based on the triangular membership (MF) which Is specified by the three parameter $\{a, b, c\}$ for each membership function $\mathrm{x}=$ (uncertain, confirmed) denoted mathematically as

$$
\text { Tirangualr }(\mathrm{x}: \mathrm{a}, \mathrm{b}, \mathrm{c})=\max \left(\min \left(\frac{x-a}{b-1}, \frac{c-x}{c-b}\right), 0\right) \ldots \ldots \ldots \ldots[12]
$$

Where parameters a,b,c determines the $\mathrm{x}$ coordinates of the three corners of the underlying triangular mem; bership function

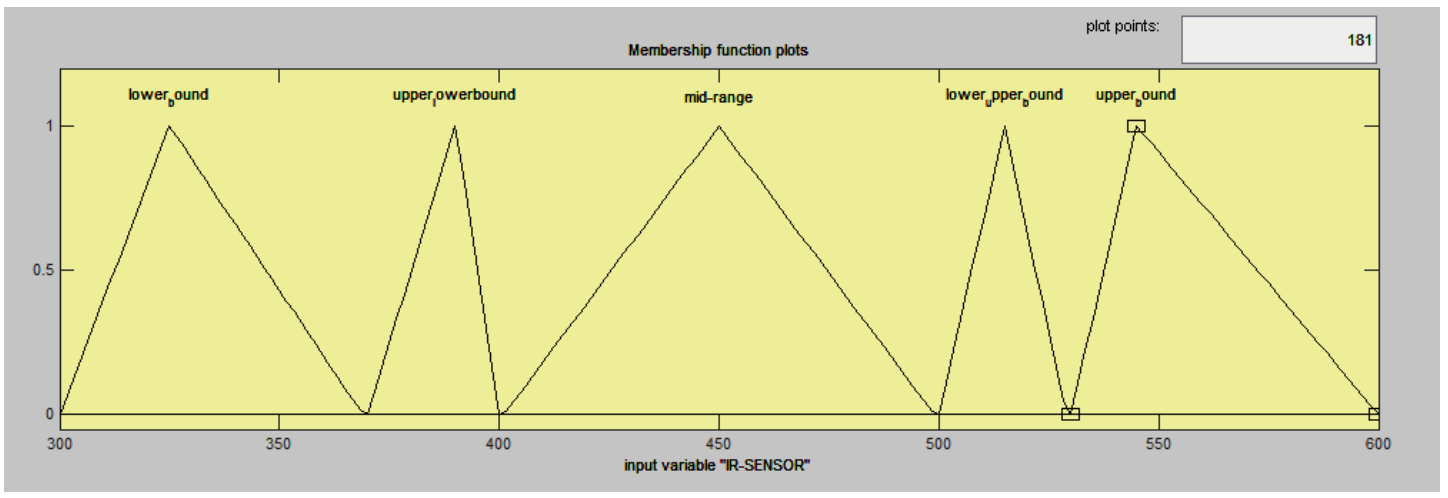

Fig.3. Fuzzy Fis Input Variable Membership Function from Matlab Fuzzy Toolbox 


\section{Output membership function}

The output variable of the fuzzy model for our proposed enhanced smart rest room automated faucet system in fig 1 above consist of five membership functions. They include slower, slow, average, fast and faster flow. Each maps to an input variables membership function. Also, they correspond to numeric values expressed in degree, since the electronic flow switch status is determined by the extent it is open, which is directly proportional to level of water free passage, as follows;

- Faster 271 to 360 degree

- Fast 181 to 270 degree

- Average 61 to 180 degree

- Slow 31 to 60 degree

- Slower 0 to 30 degree

Fig 4 below shows the output membership function for the fuzzy FIS model presented in fig1, consisting of five membership functions

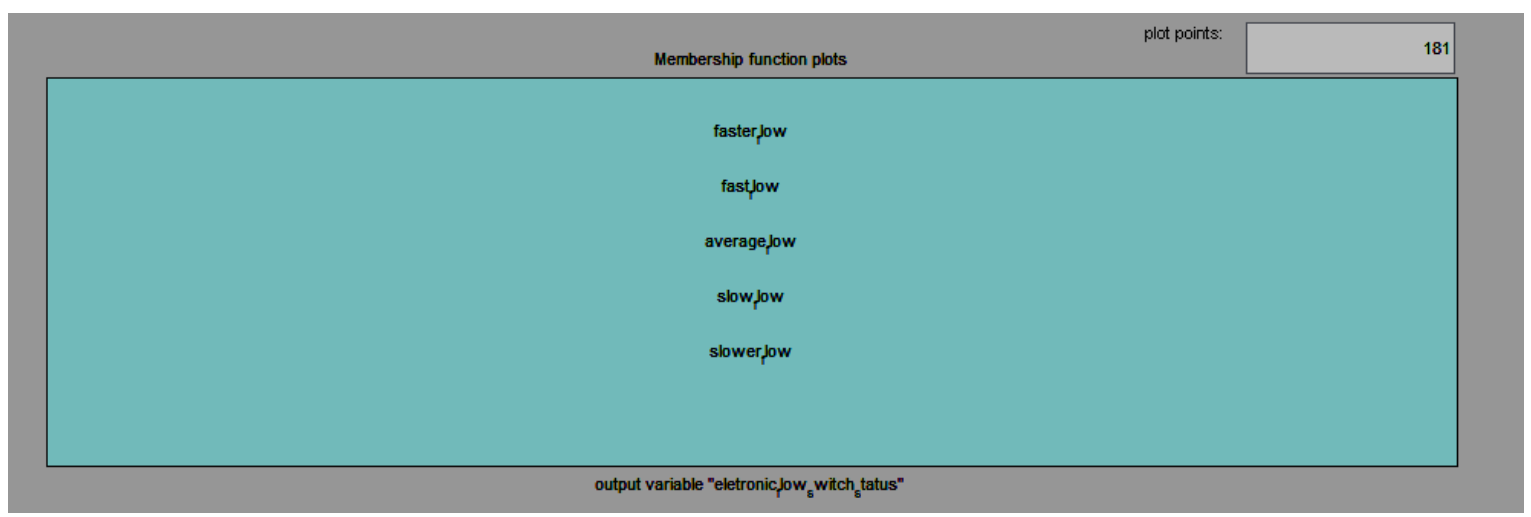

Fig.4. Proposed Smart Rest Room Automated Faucet System Fuzzy FIS Model Output Variable Membership Function

\section{System Operational Structure}

The simulated fuzzy model of the proposed enhanced smart rest room automated faucet system is shown graphically below in fig5. Derived from a graph plot of input variable membership functions on the $\mathrm{x}$-axis and output variable membership functions on the y-axis respectively. Clearly, we can infer that the range of values for input variables membership functions and their corresponding output variables membership function values in degrees are interlinked. For example lower bound on x-axis is 300 to 369 , which is also seen not to exceed 30degree on the corresponding y-axis electronic flow switch status value. Similarly for other others. Hence we can further infer that, a full implementation on commercially available smart rest room automated faucet system products, will surely conserve water resource, through adequate utilization. 


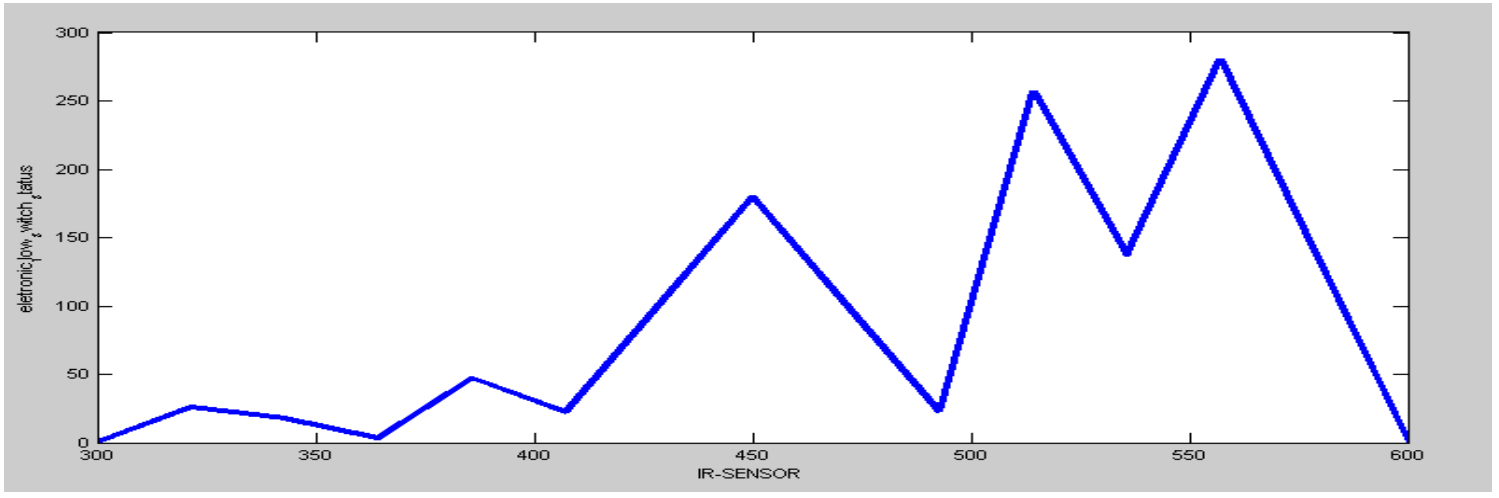

Fig.5. A Plot of Input Variable Membership Function(X) and Output Variable Membership Function(y)

\subsection{Hardware architecture}

Having simplified the proposed system operational functionality with the fuzzy model in fig1, described in the previous section. The practical implementation of the simulated model is presented in this section with the overall system hardware architecture given in figure6, depicting the components and interconnection among them. The entire system control is handled by the microcontroller, which is synornymous to a general purpose computer processor. All other components are peripherals which either acts as input (obstacle sensor)or output(electronic flow switch) unit for the system

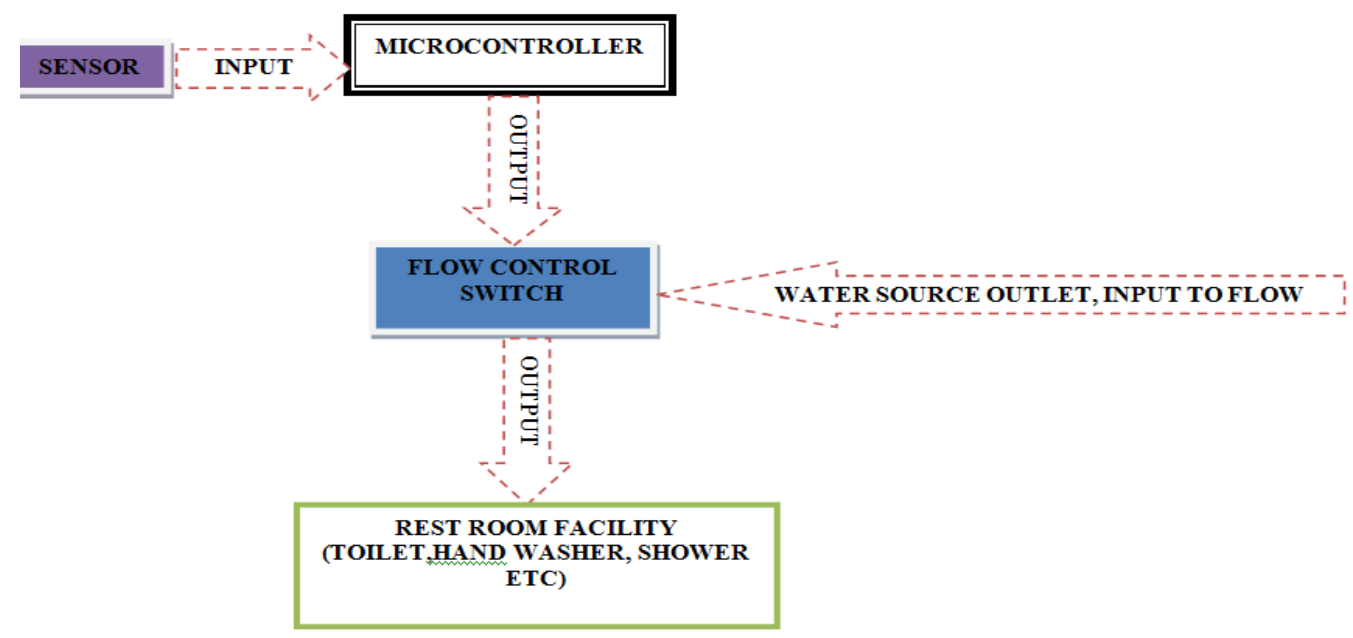

Fig.6. Architechture of Proposed Smart Rest Room Automated Faucet System

\section{A. ATMEL328 microcontroller}

The atmel328 microcontroller is the heart of the system, likened to the microprocessor of a general purpose computer system. Although with limited processing and memory capacity. The microcontroller houses the intelligent kernel that implements the fuzzy logic. The choice of this controller was influenced by the ease of interfacing and programming, operational efficiency as 16-bit controller and low cost for mass production of system for commercial use. 


\section{B. IR obstacle sensor}

This is the only input unit to the system. It detects the closeness of objects based on infrared technology, and represents it in digital signals, which are fed to the microcontroller for further processing to determine the output status

\section{Electronic water flow switch}

This component acts as the system output unit. It is an electromagnetic flow control valve, that is triggered by electronic signal from the microcontroller. By counting the pulses from the output of sensor, we can easily calculate the water flow. Each pulse is approximately 2.25 milliliters. Therefore we can mathematically express the amount of water consumed (AWC) as follows

$$
\mathrm{AWC}=\mathrm{sv} * \mathrm{wqps}
$$

Where $\mathrm{sv}=$ signal value on the electronic flow switch

wqps $=$ water quantity per signal, a constant supplied from the manufacturer data sheet

\subsection{Operational flowchart of the smart rest room automated faucet system}

The flowchart below is a diagrammatic illustration of the operational sequence and structure of the enhanced smart rest room automated faucet system. First, the system initializes, thereafter the obstacle sensor inputs it's reading(values) to the system. This is checked for validity of specified electronic flow switch activation range values. Once a valid range is detected, the electronic flow switch is activated base on the range detected corresponding degree of opening. Since rate of water flow which is directly proptional to the signal level on the electronic flow switch. Else if no valid range is detected, the system returns to continue monitoring input values from the obstacle sensor until the system gets a valid input value that falls within a range.

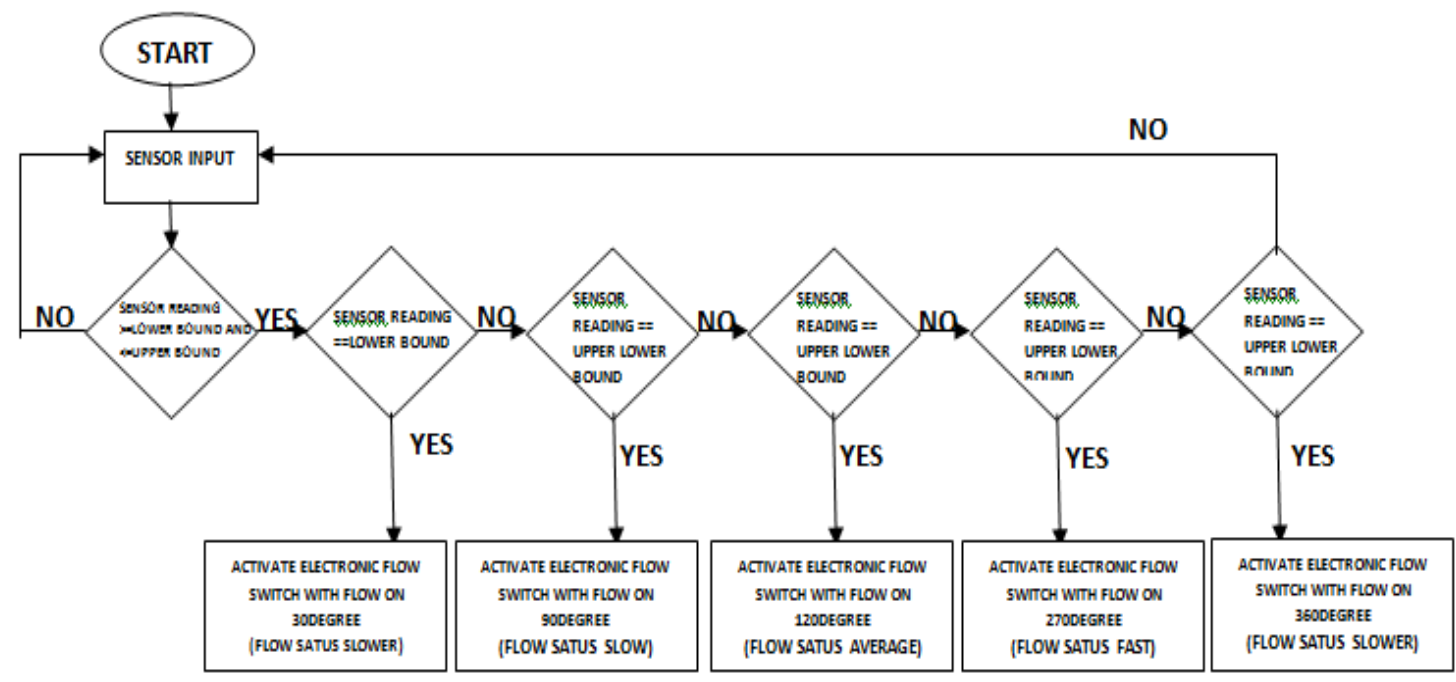

Fig.7. Operational Flowchart of the Proposed Automated Smart Rest Room Automated Faucet System 


\section{System Implementation and Data Analysis}

During the system implementation, the infrared obstacle sensor was calibrated to activate the flow switch on 300 to 600 value range on a $3 \mathrm{~cm}$ distance. This range is further divided according to fuzzy model into five sub ranges mapped on numerical values 300-369, 370-400, $401-499,500-529$ and $530-600$, corresponding to lower bound, upper lowerbound, mid-range, lower upper bound and upper bound respectively.

Data logged was analysed to further understand the system operation and performance as follows

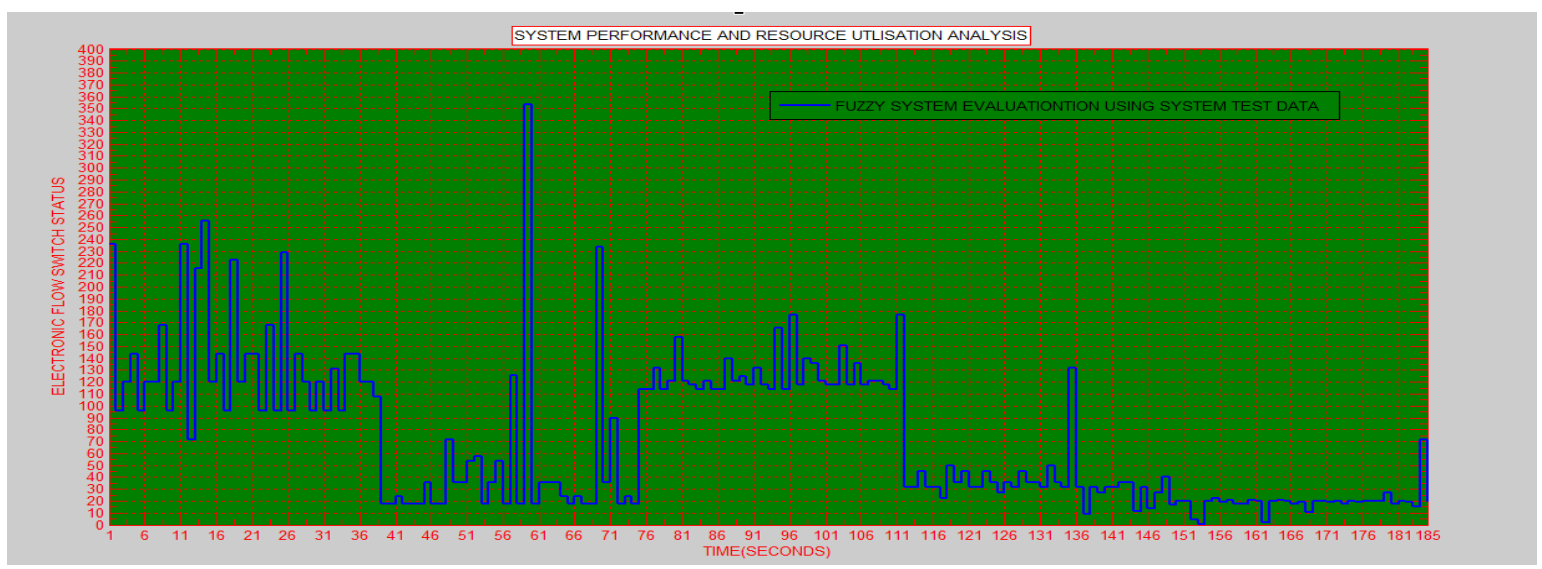

Fig.8. System Performance and Operational Analysis of Electronic Flow Switch State

\subsection{Performance evaluation}

Fig8 above represents the output of the system performance during an evaluation of our simulated fuzzy model using data logged from practical implementation of the system under a 3 minutes and 5seconds test operation, we can infer as follow;

Clearly from the first seconds to 38 seconds, we can infer that water flow rate which is directly related to the opeining rate of the electronic flow switch, although undulates between fast, and average, but was mostly average at time intervals 2 to 11 seconds, 15 to 17 seconds, $19-25$ seconds and 26 to 38 seconds. With electronic water flow switch valve opening up to above $91^{\circ}$ and $180^{\circ}$ most commonly. Except for few seconds where its below $90^{\circ}$ corresponding to slow flow rate at time interval 12 seconds. And above $180^{\circ}$ (between $181^{\circ}$ to $258^{\circ}$ ) at time interval 1 seconds, 11.5 seconds, 13 to 14 seconds, 18 seconds, and 25.5 seconds.

Also, from the $39^{\text {th }}$ seconds through to the $76^{\text {th }}$ seconds, we can infer that water flow rate was mostly slow, with electronic water flow switch valve opening up to above $30^{\circ}$ and $120^{\circ}$ most commonly, except for few seconds where its below 30o and above 120o. consequently affecting the flow rate of water as well to fast (69seconds59seconds, and 57.30seconds)and slower(39 to 40seconds, 42 to 44 seconds, 47 to 48.50 second, 52.50seconds, 57seconds, 58seconds, 60seconds, 68seconds, 72seconds, and 74.50seconds) respectively.

Furthermore, observation from the 77 seconds up till the 111 seconds from fig8 above, clearly water flow rate is within the average, with the electronic flow switch valve opening up to between $120^{\circ}$ to $170^{\circ}$ most commonly, except for a few seconds where it's below $120^{\circ}$ (78seconds, 82 to 83 seconds, 85 to 87 seconds, 90seconds, 93seconds,95seconds, 98seconds, 102seconds,104seconds, 107seconds, and 110seconds)

In addition, from the 113 seconds to the $144^{\text {th }}$ seconds in the 3 minutes 5 secinds test of the system as observed from fig8 above, the water flow rate varies within the slow flow rate, where the electronic flow switch valve opens up to between $30^{\circ}$ to $50^{\circ}$ most commonly, except for some instances where it is $30^{\circ}$ and below(112seconds, 115 to 116 seconds, 117 seconds, 125 seconds, 137 seconds, 144 seconds) as well as above $50^{\circ}$ (135seconds) 
Further observation of the non-linear operation of the system from above, we can also notice that from the 146 seconds till the last seconds of the 3 minutes 5 seconds test, the water flow rate was slower, with electronic flow switch opening up to mostly $30^{\circ}$ and below, except for some few seconds where its slightly pushed into slower flow rate to slow flow rate, with electronic flow switch opening up to $40^{\circ}$ and $70^{\circ}$ at 148 seconds and 185 seconds respectively.

\subsection{Water consumtpion}

The water consumption estimation was obtained using the eq(2) in conjunction with each signal value input to the electronic flow switch from the embedded microcontroller. Fig9 below represent the corresponding electronic flow switch activation states of fig8 above, indicating the amount of water expended at each stage in milliliters. The highest quantity of water expended is at the $57^{\text {th }}$ to $58^{\text {th }}$ seconds of fig8 above corresponding to 790 milliliters, as seen in fig9 below. This corresponds to same faster water flow rate at electronic flow switch value, opening up to $356^{\circ}$ in fig8 above. Similarly for slower water flow rate in fig8 above with electronic flow switch valve opening up to $30^{\circ}$ maximum, clearly implies a very small amount of water being released and used as in fig9 below. Furthermore, from fig9 below it is obvious that Slower, slow, average, fast and faster water flow rate uses 40 to 60,70 to 200,220 to 400,390 to 570 and 571 and above milliliters of water per signal level respectively.

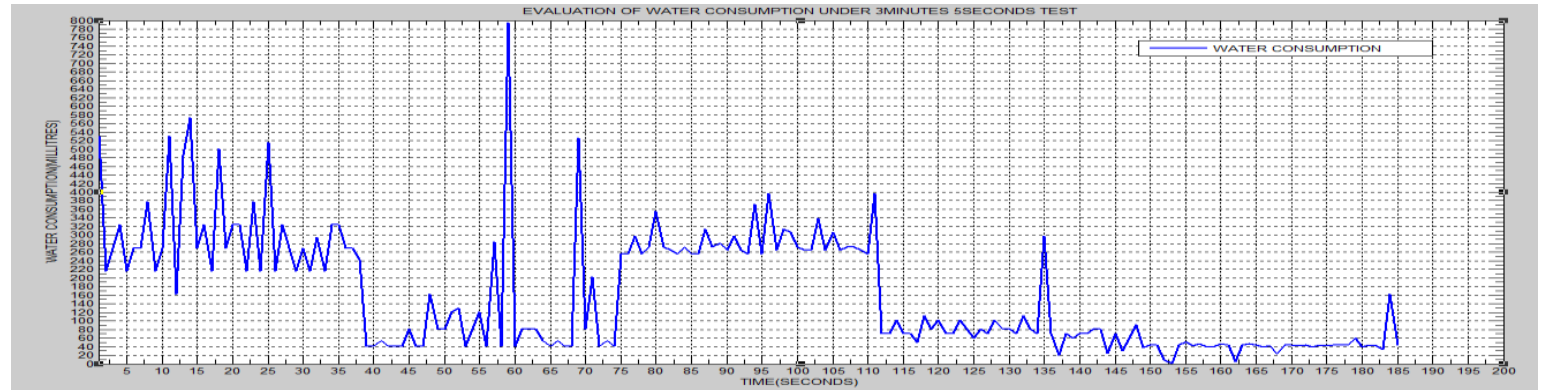

Fig.9. Water Consumption Analysis

\section{Result}

From the above data analysis it is clear that traditional ON/OFF smart rest room automated faucet systems with crisp boundaries are prone to the following pitfalls

$>$ Wastage of water resource, due to full activation of electronic flow switch once lower bound sensor value is detected, and water flows at high speed.

$>$ Also, energy consumption is another challenge for existing smart rest room automated faucet systems, which activate electronic flow switch completely with full energy.

Hence from this prototype model which enhances existing smart rest room automated faucet system, through the removal of crisp boundary for activation of water flow level with the creation of five subgroups boundaries that will activate the flow switch and consequently approximate the quantity of water needed, by controlling the water flow rate effectively, limits water resource wastage and energy usage. As we can clearly infer from fig8 above faster water flow rate and fast water flow rate were rare, which implies that less amount of water is used in by the system following its corresponding water consumption in fig9. Also the object position within the activation boundary values (lower and upper bound) determines the level of water flow. Therefore, a situation where you just need a few drops on your hand from a hand wash basin equipped with automated 
faucet system, approximately same amount of water will be released in relation to the electronic flow switch valve opening and same will be utilized.

\section{Conclusion}

An advance smart rest room automated faucet system was developed, based on a fuzzy logic model designed in this paper, which help to eliminate the shortcoming of existing automated faucets system that operates on traditional control mechanism having crisp boundaries. A fuzzy model of the prototype system was first developed, to test the feasibility of the proposed system. After which, an embedded hardware software solution was developed and tested. During the test, data collected was analyzed and the results clearly indicates the efficiency of the fuzzy based smart rest room automated faucet system,. Utilizing water efficiently, compared with others implemented with traditional control mechanism with crisp activation boundary in terms of water resource utilization and effective control of water release. This was achieved through data analysis which helped to understand the water consumption rate and the various states of the electronic flow switch valve during the system implementation. This system is no doubt an enhancement to existing smart rest room automated faucet system, with promise of improved performance and water resource utilization.

\section{References}

[1] Trupti Wani, Mohan Raj, Tauqeer Raza, Noble K V., Design and Development of Automated Faucet Valve Regulating Mechanism, ijesrt international journal of engineering sciences \& research technology Vol 3(8): August, 2014, pp795-803.

[2] Aaron Bianchi David Markham, Joseph Pasquarelli Jaimes Spring, All-In-One Hand Washing System, Project Number: MQP-MQF 3115, worchester polytechnic institute MIRAD Laboratory, April 26, 201.

[3] Paul PANEK, Håkan NEVERYD, Wolfgang L. ZAGLER, Developing a More UserFriendly Rest Room, Reprint from: Proceedings of the AAATE Conference 2003, Assistive Technology - Shaping the Future, Ger M. Craddock, Lisa P. McCormack, Richard B. Reilly, Harry T.P. Knops (eds.) August 30-September 03, 2003, Dublin, Ireland, pp.678-682.

[4] Ranjay Krishna, Eunae Cho, Benjamin Jaeger Interactive Faucet: Final Report, Fun Lavoratories November 30, 2012.

[5] Ria Sood, Manjit Kaur, Hemant Lenka, Design and development of automatic Water flowmeter International Journal of Computer Science, Engineering and Applications (IJCSEA) Vol.3, No.3, June 2013, DOI: $10.5121 /$ ijcsea.2013.3306 49.

[6] C.Sugitha, Fleena Christy, S.Chandrasekaran, Intelligent Machine Learning System For Smart Room Using Sensor Network International Journal of Innovative Research in Computer and Communication Engineering, Vol. 1, Issue 6, August 2013 PP1328-1335.

[7] Jehad Al Dallal, Anas Abdin, Dalal Al Hulailah, Hawra Al Khamis, Wadha Al Ghanim Integrated Hardware and Software System for Controlling Water Consumption ARPN Journal of Systems and Software VOL. 4, NO. 1, January 2014 pp 1-4.

[8] Mohamed aamir. M, kamalanathan.p.. Automatic urinal flushing system, International Journal of Science, Engineering and Technology Research (IJSETR), Volume 4, Issue 4, April 2015.

[9] Raghied Mohammed Atta Purity Sensor Activated Smart Toilet Flushing System, International Journal of Water Resources and Arid Environments 2(1): 51-55, 2013.

[10] 11florida rural water resource association, "automatic flushing valve; build your own automatic flushing valve" (pdf) accessed 14/03/2016.

[11] 12Rosslin John Robles and Tai-hoon Kim, Applications, Systems and Methods in Smart Home Technology: A Review, International Journal of Advanced Science and Technology Vol. 15, February, $2010 \mathrm{pp} 37-48$. 
[12] Jyh-sing roger jang, chuen-tsai.sun, and eijl mizutani, Neurofuzzy and soft computing: A computational approach to learning and machine intelligence, Eastern Economy Edition.

\section{Authors' Profiles}

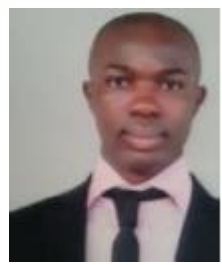

Ighoyota B. Ajenaghughrure, recently his masters in computer science from Vels University India, in 2016 may. While he obtained his bachelor's in computer science from Delta State University. His research interest are in artificial intelligence, Human computer Interaction, health and business information systems, Trust and security computing, and computer vision application.

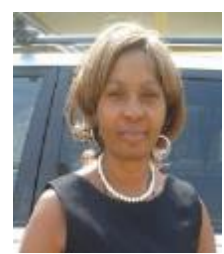

Dr. Maureen I. akazue is a Lecturer in the Department of Mathematics \& Computer Science, Delta State University, Abraka, Delta State, Nigeria. She received Master of Information Science degree in 2001 from University of Ibadan, Oyo State, Nigeria, M.Sc.Computer Science in 2008 and PhD Computer Science in 2014, both from University of Benin, Edo State, Nigeria. Her research interests are HCI, Enhancing Customer's satisfaction, Online fraud prevention, Security challenges and Computer Solutions, business information systems, and Trust computing

How to cite this paper: Akazue I. Maureen, Ajenaghughrure Ighoyota Ben,"Fuzzy Based Enhanced Smart Rest Room Automated Faucet System", International Journal of Engineering and Manufacturing(IJEM), Vol.7, No.3, pp.20-30, 2017.DOI: 10.5815/ijem.2017.03.03 